\title{
Single and double electron photodetachment from the oxygen anion at $41.7 \mathrm{~nm}$
}

\author{
L. S. Harbo, ${ }^{1}$ A. Becker ${ }^{2}$ S. Dziarzhytski, ${ }^{3}$ C. Domesle, ${ }^{2}$ N. Guerassimova, ${ }^{3}$ A. Wolf, ${ }^{2}$ and H. B. Pedersen ${ }^{1, *}$ \\ ${ }^{1}$ Department of Physics and Astronomy, Aarhus University, DK-8000 Aarhus C, Denmark \\ ${ }^{2}$ Max-Planck-Institut für Kernphysik, D-69117 Heidelberg, Germany \\ ${ }^{3}$ HASYLAB at DESY, Hamburg, Germany
}

(Received 24 June 2012; published 14 August 2012)

\begin{abstract}
Single and double photodetachment of the oxygen anion $\mathrm{O}^{-}$have been investigated at $41.7 \mathrm{~nm}(29.8 \mathrm{eV})$ in a crossed beams experiment using intense photon pulses from a free-electron laser. The ratio of single $\left(\mathrm{O}^{0}+e^{-}\right)$ and double $\left(\mathrm{O}^{+}+2 e^{-}\right)$detachment was determined to be $\sigma_{\mathrm{O}^{0}} / \sigma_{\mathrm{O}^{+}}=4.12 \pm 0.17$ as identified directly from the yield of $\mathrm{O}^{0}$ and $\mathrm{O}^{+}$fragments after irradiation. The absolute cross section for the dominating single detachment channel was measured to $\sigma_{\mathrm{O}^{0}}=(2.1 \pm 0.6) \times 10^{-19} \mathrm{~cm}^{2}$. Analysis of photoelectrons detected in coincidence with neutral fragments $\left(\mathrm{O}^{0}\right)$ suggests that single photodetachment primarily happens via the ground $\left({ }^{3} P\right)$ or lowest excited $\left({ }^{1} D\right)$ state of oxygen. The results demonstrate the feasibility and advantage of crossed beams experiments for complete studies of photodetachment reactions.
\end{abstract}

DOI: 10.1103/PhysRevA.86.023409

PACS number(s): $32.80 . \mathrm{Gc}$

\section{INTRODUCTION}

Studies of electron detachment processes from negative ions $\left(A^{-}\right)$, shortly written as

$$
A^{-} \stackrel{c}{\rightarrow} A^{n-1}+n e^{-},
$$

have provided some of the most detailed insight into the structure and dynamics of atomic ions [1]. Single $(n=1)$ or multiple $(n \geqslant 2)$ electron detachments reactions have been induced both by collisions with heavy targets ( $c=$ gas) [2], with electrons $\left(c=e^{-}\right)[3,4]$, and by photoabsorption $(c=\gamma)[1]$.

Single electron photodetachment $(n=1)$ of atomic anions has been studied extensively in the infrared and ultraviolet regime using lasers, see, e.g., Ref. [1], where properties of both the emerging neutral atoms and the emitted photoelectrons have been analyzed. Investigations of single electron photodetachment in the extreme ultraviolet (xuv) regime were pioneered with Doppler-tuned spectroscopy on relativistic ion beams for the case of the atomic hydrogen anion $\left(\mathrm{H}^{-}\right.$and $\left.\mathrm{D}^{-}\right)$ [5-7]. Experimental studies on double electron photodetachment $(n=2)$ reactions were also first explored with the combination of lasers and relativistic ion beams for $\mathrm{H}^{-}$[8], followed by experiments with fast (nonrelativistic) ion beams and lasers for $\mathrm{He}^{-}$[9] and $\mathrm{K}^{-}$[10], where the focus was on characterizing the threshold region still accessible with ultraviolet (uv) lasers.

Within the last decade more universal experimental setups $[11,12]$ for detachment and ionization experiments of ionic targets in the xuv range have been realized, where intense synchrotron radiation is merged co-linearly with fast moving ion beams. With these experimental systems, benchmarking results of wavelength resolved absolute cross sections for double electron photodetachment have been obtained both for cases of inner-shell and valence-shell electron detachment [11-19]. Several cases of multiple electron photodetachment $(n \geqslant 3)$ have also been investigated [20-23].

For photon energy regimes where inner-shell detachments is possible, e.g., studies of $\mathrm{He}^{-}$[14], $\mathrm{Li}^{-}$[12,13], $\mathrm{C}^{-}$[15],

\footnotetext{
*Author to whom correspondence should be addressed: hbjp@phys.au.dk
}

and $\mathrm{S}^{-}$[22], the double detachment reaction mostly seems to proceed as a sequential process of inner-shell electron detachment followed by Auger decay showing, however, also delicate processes near thresholds such as the postcollisional re-capture [19,24] and the double Auger decay from a hollow resonance [18]. In contrast, for photon energy regions where valence electrons are accessed, e.g., experiments on $\mathrm{Na}^{-}$[11], $\mathrm{F}^{-}$[16], and $\mathrm{Cl}^{-}$[17], double detachment mechanisms both of indirect (sequential) nature, involving detachment into excited neutrals states followed by autoionization, and of direct nature with simultaneous emission of two electrons have been discussed. In particular, for the halogen anions $\mathrm{F}^{-}$[16] and $\mathrm{Cl}^{-}$[17] simultaneous processes are believed to dominate over sequential processes.

The experimental systems using merged beams [11,12] are limited in the sense that they inherently are unable to observe neither neutral fragments that originate from the single electron detachment, nor emerging photoelectrons. For instance, techniques developed to detect neutral fragments in a merged beams setup in lower photon energy regimes, see, e.g., Ref. [25], are not extendable to high photon energies, and the use of inclined beams geometries have so far not been reported. Moreover, especially photoelectron spectroscopy in the present merged beams setups seems difficult to realize.

Evidently, these experimental limitations also restrict the conclusions that can be drawn from the experimental data without additional theoretical interpretation. For example, an important outstanding issue in the xuv regime is the significance of single detachment $(n=1)$ in comparison to the double (and multiple) detachment $(n \geqslant 2)$ processes. This is true both for processes involving K-shell detachment, where for instance the postcollisional recapture processes [19,24] could be more directly characterized by observation of neutral fragments, as well as for processes involving detachment of valence electrons, where the ratio of single to double detachment has only been roughly estimated for $\mathrm{He}^{-}$[9]. Moreover, analysis of the properties of the emitted photoelectrons would evidently ease the experimental assignment of detachment mechanisms as dominated by sequential or simultaneous electron emissions. 


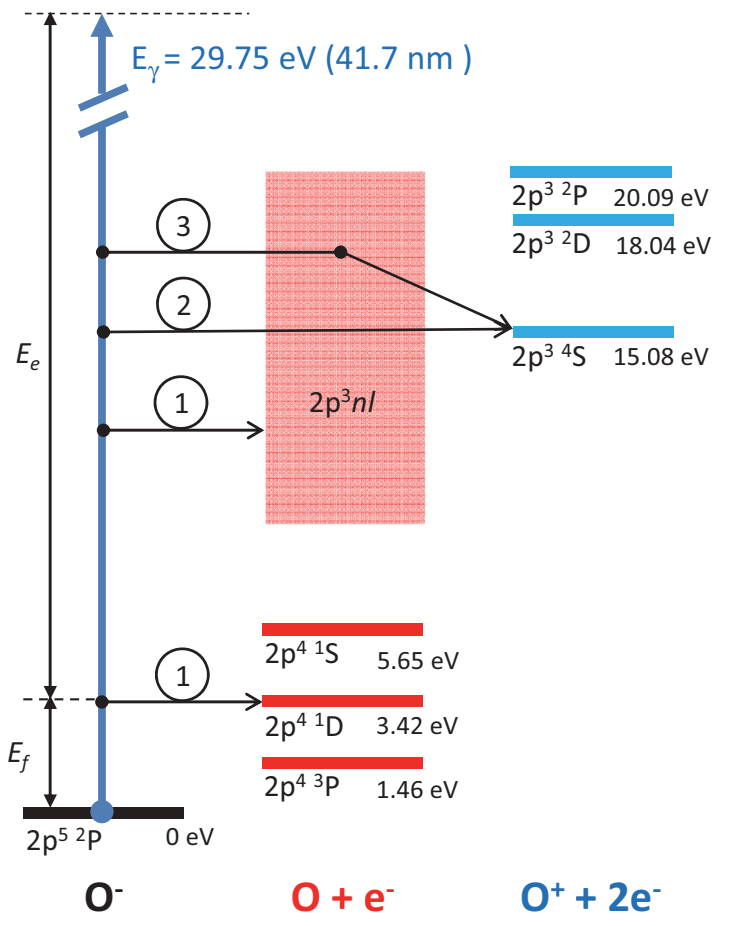

FIG. 1. (Color online) Schematic energy-level diagram for $\mathrm{O}^{-}$, $\mathrm{O}^{0}$, and $\mathrm{O}^{+}$in the region accessible by $41.7-\mathrm{nm}$ photoabsorption and illustration of electron photodetachment processes. Processes labeled by 1 show single detachments while processes labeled 2 and 3 show direct (simultaneous) and indirect (sequential) double photodetachment, respectively.

In this paper, we address these two experimental limitations with an exploratory study of the oxygen anion at $41.7 \mathrm{~nm}$ ( $29.8 \mathrm{eV}$ ) irradiation using a crossed beams setup in combination with an intense free electron laser (FEL). We focus in this paper mainly on the characterization of the ratio of single and double photodetachment processes; however, we also discuss the possibilities for electron spectroscopy in the crossed beams geometry.

Numerous aspects of the photodetachment of the oxygen anion have been studied previously in the ir and uv regimes, yielding the ground state $\left({ }^{3} \mathrm{P}\right)$ of the oxygen $\left(\mathrm{O}^{0}\right)$ as product; see, e.g., Ref. [26] for a recent overview of literature. Figure 1 shows schematically the energy levels of $\mathrm{O}^{0}$ and $\mathrm{O}^{+}$and processes that are accessible with the 41.7-nm radiation used in this work. Thus, under $41.7 \mathrm{~nm}\left(E_{\gamma}=29.8 \mathrm{eV}\right)$ irradiation, the oxygen anion can undergo single photodetachment (processes indicated by 1 in Fig. 1) leaving the $\mathrm{O}^{0}$ atom in its ground or excited states while emitting a photoelectron with energy $E_{e}=E_{\gamma}-E_{f}$, where $E_{f}$ is the energy of the $\mathrm{O}^{0}$ above the ground state of the anion. Double electron photodetachment can proceed as a direct process (labeled 2) with simultaneous emission of two electrons or as an indirect(sequential) process (labeled 3) involving initial single detachment followed by autoionization from an excited state of the neutral oxygen.

While previous measurements on xuv photodetachment of valence electrons $[11,16,17]$ have emphasized the importance of the double detachment channels, we demonstrate in the present work for the case of the oxygen anion that the single detachment channel dominates over double detachment by a factor of $\sim 4$.

\section{CROSSED BEAMS EXPERIMENT}

The experiment was performed at the Plane Grating monochromator (PG2) beam line [27] of the Free Electron Laser in Hamburg (FLASH) facility [28,29] at Deutsches Elektronen Syncrotron (DESY) using the ion beam facility TIFF [30] (trapped ion fragmentation with a FEL) which is installed with a crossed ion-photon interaction zone $\sim 1.5 \mathrm{~m}$ before the focus of the photon beam line.

Negative ions were produced in a hollow cathode ion source operated at a high potential with a gas inlet of pure water vapor of an approximate pressure of $0.1 \mathrm{mbar}$ and sustaining a discharge of $775 \mathrm{~V}$ and $55 \mathrm{~mA}$. From the source, ions were extracted to ground potential thereby generating a collimated beam of negative ions of kinetic energy $E_{0}=3.0 \mathrm{kV}$. The beam was subsequently mass analyzed in a magnetic field, giving a total current of oxygen anions of a few $\mathrm{nA}$ which was sufficient for the present experiment.

The mass selected $\mathrm{O}^{-}$ions were then electrically focused and guided as well as further collimated by two sets of slits. They entered the crossed beam interaction zone as a horizontal, essentially parallel beam of $I_{O^{-}}=1.0 \mathrm{nA}$, corresponding to a linear density of $l_{O^{-}}=3.3 \times 10^{2} \mathrm{~cm}^{-1}$. Figure 2 shows a schematic drawing of the present interaction zone and detector setup at TIFF. During the measurement, the ion beam was chopped [30] into 2.5- $\mu$ s-long pulses, matched to the time structure of FLASH. After the interaction region the ion pulses were bent upwards and dumped.

For the present experiment the FLASH facility delivered intense horizontally polarized radiation at a wavelength of $41.7 \pm 0.5 \mathrm{~nm}$ with a repetition rate of $10 \mathrm{~Hz}$ in the form of trains of 50 short $(\sim 250 \mathrm{fs}$ ) pulses with a time interval of $5 \mu \mathrm{s}$ between them. The average pulse intensity after spatial collimation was $30 \mu \mathrm{J} /$ pulse, i.e., $N_{\gamma}=6 \times 10^{12}$ photons/pulse. The transmission through the PG2 beam line was $T_{P G 2}=$ $0.5 \pm 0.1$, thus resulting in an average of $15 \mu \mathrm{J} /$ pulse in the interaction zone. Here the photon and ion pulses were crossed at $90^{\circ}$ inside a newly implemented photoelectron spectrometer (see Fig. 2). Before and after the interaction region the photon beam was carefully aligned through slits of $3 \times 3$-mm openings, which also prevented most of the stray light generated in the PG2 beam line from entering the electron spectrometer and detector region. About $3 \mathrm{~m}$ downstream from the interaction region, the photon pulses were sent onto a $\mathrm{Cu}$ plate biased to $-800 \mathrm{~V}$ from which both a precise timing signal for the arrival of the photon pulses as well as a signal proportional to pulse energy were derived.

The vertical intensity profiles of the two beams were monitored by moving inside the electron spectrometer a 1-mm-wide needle across the two beams while measuring the ion current and the pulse height of the amplified and shaped signal extracted from the biased $\mathrm{Cu}$ plate. The beam profiles determined in this way are shown in Fig. 2. Considering the convolution of the measured intensity distributions with the width of the needle, the vertical profiles of both beams can be well approximated by Gaussian distributions with, in the present case, very similar widths of $0.39 \mathrm{~mm}(\mathrm{rms})$. The 


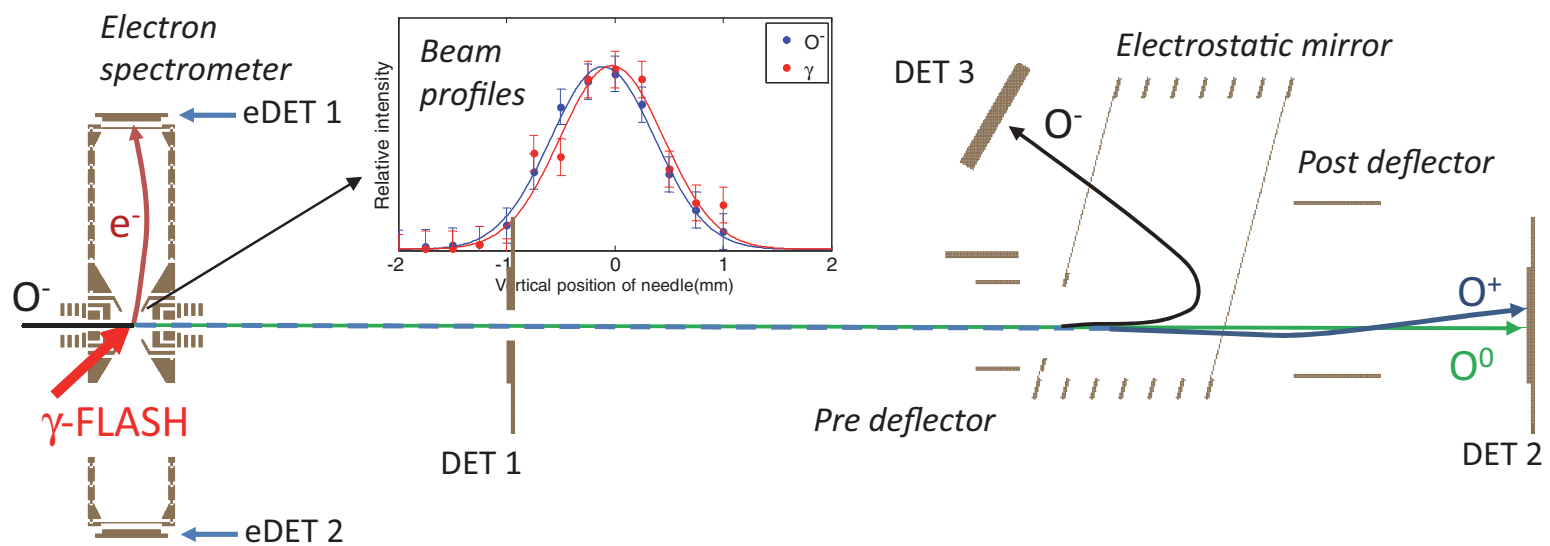

FIG. 2. (Color online) The experimental setup around the interaction region of the TIFF experimental setup [30] at FLASH [28,29]. The lines show calculated fragment trajectories for $\mathrm{O}^{0}$ (green) and $\mathrm{O}^{+}$(blue) after photodetachment in the interaction zone. The inset shows the vertical profiles of the two beams as measured with the 1-mm insertable needle in the middle of the interaction region.

beam centers coincide with a remaining shift of $0.02 \mathrm{~mm}$. In consequence the vertical overlap factor [30] can be determined to be $F=7.3 \pm 0.1 \mathrm{~cm}^{-1}$.

As shown in Fig. 2, the TIFF experiment involves three position and time resolving detectors for atomic and molecular fragments (DET 1-3) and two detectors for photoelectrons (eDET1-2). The experiment also involves an electrostatic mirror that is normally used to separate heavy charge fragments from the original ion beam.

In this exploratory experiment we used only DET 2 for detection of both neutral $\mathrm{O}^{0}$ atoms and single charged $\mathrm{O}^{+}$ ions. The setup around the electrostatic mirror was actively used to deflect the main ion beam $\left(\mathrm{O}^{-}\right)$towards DET 3 as well as to advance and deflect the $\mathrm{O}^{+}$ion relative to the neutral $\mathrm{O}^{0}$. Following the deflection of the anions in the electrostatic mirror, $\mathrm{O}^{+}$and $\mathrm{O}^{0}$ could be distinguished from each other on DET 2 by their different positions of impact. Moreover, the applied potentials lead to a shorter time of flight (TOF) for the ions $\left(\mathrm{O}^{+}\right)$from the $\mathrm{O}^{-}$photodetachment than for the neutrals $\left(\mathrm{O}^{0}\right)$. To ensure the same efficiency for detection of the $\mathrm{O}^{0}$ and $\mathrm{O}^{+}$fragments the stack of multichannel plates of DET 2 was biased with ground potential on its front.

During the experiment the data collection was continuously (at twice the FLASH repetition rate, i.e., at $20 \mathrm{~Hz}$ ) alternated between four different conditions, namely with (1) both ions and photon pulses in the interaction region, (2) only ion pulses, (3) only photon pulses, and (4) neither ion nor photon pulses (dark counts). To reduce the background from processes in the residual gas, the pressure in the interaction region was kept at ultrahigh vacuum of $1-3 \times 10^{-10}$ mbar, during the measurement.

\section{RESULTS}

\section{A. Ratio of single and double detachment}

Figure 3(a) displays the observed distributions of TOFs for particles impacting on DET 2 as referenced to the photon arrival time. A major background stems from single and double detachment from $\mathrm{O}^{-}$by the residual gas of the vacuum chamber (red curve, ions only). By changing the deflection field just before DET 2, using the postdeflector shown in
Fig. 2, it was verified that double detachment (yielding $\mathrm{O}^{+}$) dominates this background. A contribution to the background is also observed when only photon pulses are present in the interaction region (black curve); this signal $(\gamma)$ stems from a small fraction of photons scattered to DET 2 at the subsequent FLASH pulse. The fragments from single $\left(\mathrm{O}^{0}\right)$ and double $\left(\mathrm{O}^{+}\right)$photodetachment are identified as narrow peaks at 4880 and $5150 \mathrm{~ns}$, respectively; the width of these peaks $(\sim 5 \mathrm{~ns})$ reflects a spread of $\sim 0.1 \%$ in the ion-beam energy.

Figure $3(\mathrm{~b})$ shows the spatial positions of impact on DET 2 for the events in the TOF regions around the photoinduced $\mathrm{O}^{0}$ and $\mathrm{O}^{+}$fragments. Two small, separate, and well defined regions of the detector surface are seen to accept impacts of the $\mathrm{O}^{0}$ and $\mathrm{O}^{+}$fragments produced in photodetachment, also demonstrating that DET 2 indeed accepts all photoinduced fragments. In Fig. 3(c) the resulting TOF distribution is shown after selecting events with impact positions in narrow spatial regions around the impact positions of $\mathrm{O}^{0}$ and $\mathrm{O}^{+}$in Fig. 3(b), where also the remaining (small) background from residual gas interactions, stray photons, and dark counts has been subtracted.

The number of detected particles $N_{X}$ for either single ( $X=$ $\left.O^{0}\right)$ or double $\left(X=O^{+}\right)$detachment can be written as

$$
N_{X}=\sigma_{X}\left\langle N_{\gamma}\right\rangle T_{P G 2} P_{\gamma} l_{O^{-}} F \varepsilon_{X},
$$

where $\sigma_{X}$ is the reaction cross section, $\left\langle N_{\gamma}\right\rangle$ is the average number of photons per pulse, $P_{\gamma}$ is the number of photonion crossings, $l_{O^{-}}$is the linear ion density, $F$ is the spatial overlap factor, and $\varepsilon_{X}$ is the detector efficiency for particle of type $X$. With the values for the present experiment, and assuming a detector efficiency of $\varepsilon_{O^{0}}=0.5 \pm 0.1$, this results in a cross section of $\sigma_{O^{0}}=(2.1 \pm 0.6) \times 10^{-19} \mathrm{~cm}^{2}$ for the single detachment channel.

Since the two types of fragments $\left(\mathrm{O}^{0}\right.$ and $\left.\mathrm{O}^{+}\right)$are detected with the same efficiency, $\varepsilon_{O^{0}}=\varepsilon_{O^{+}}$, the ratio of single and double detachment can be directly obtained from the ratio of counts in the two peaks shown in Fig. 3(c). (For this figure it amounts to $\left.\sigma_{\mathrm{O}^{0}} / \sigma_{\mathrm{O}^{+}}=3.99 \pm 0.26\right)$. To investigate a possible variation of the detector efficiency depending on the spatial location of particle impact on DET 2, i.e., $\varepsilon_{O^{0}, O^{+}}=\varepsilon_{O^{0}, O^{+}}(x, y)$, measurements were made for different 

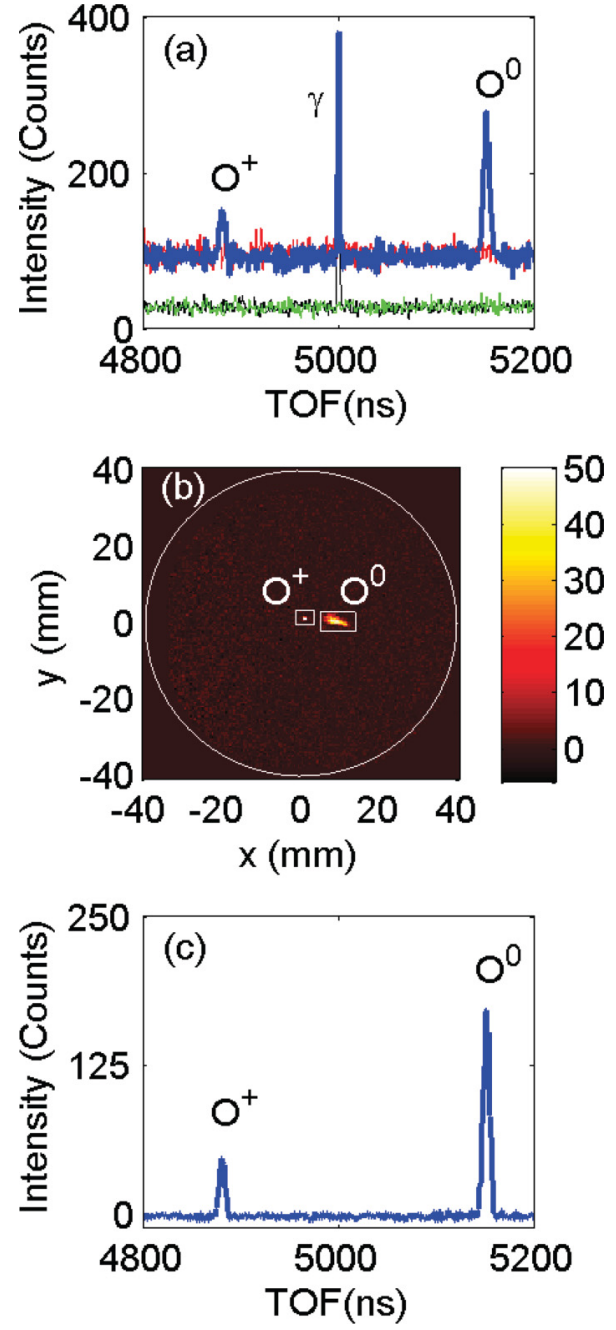

FIG. 3. (Color online) Detection of $\mathrm{O}^{0}$ and $\mathrm{O}^{+}$fragments on DET 2 as obtained after $1.3 \times 10^{6}$ ion-photon crossings. (a) Distribution of time of flight (TOF) relative to the FLASH arrival, as observed with DET 2. The traces representing different conditions of measurement are explained in the text. The $\mathrm{O}^{+}$ions formed in double detachment are seen as a narrow peak at $4880 \mathrm{~ns}$ in the distribution obtained with both photons and ions in the interaction region. Similarly, the neutral oxygen atoms $\left(\mathrm{O}^{0}\right)$ from single detachment appears at $5150 \mathrm{~ns}$, while the peak labeled $\gamma$ represents a photon background (see the text). (b) Distribution of particle impact positions on DET 2 for $4870 \mathrm{~ns} \leqslant$ TOF $\leqslant 4991 \mathrm{~ns}\left(\mathrm{O}^{+}\right)$or for $5140 \mathrm{~ns} \leqslant \mathrm{TOF} \leqslant 5160 \mathrm{~ns}\left(\mathrm{O}^{0}\right)$. (c) TOF distribution from DET 2 for impact positions near the regions of $\mathrm{O}^{0}$ and $\mathrm{O}^{+}$seen in (b).

mean impact positions of the $\mathrm{O}^{+}$fragment. Thus, the spatial position of the $\mathrm{O}^{+}$particles was varied using the postdeflectors seen in Fig. 2. The result of this investigation is shown in Fig. 4 where the ratio $\sigma_{\mathrm{O}^{+}} / \sigma_{\mathrm{O}^{0}}$ is plotted versus the mean spatial separation of detected $\mathrm{O}^{+}$and $\mathrm{O}^{0}$ particles. Evidently, there is no significant variation of the detector efficiency across DET 2. Combining all data, we obtain as the final experimental value for the ratio of double and single detachment cross section for $\mathrm{O}^{-}$at $41.7 \mathrm{~nm}$,

$$
\frac{\sigma_{\mathrm{O}^{0}}}{\sigma_{\mathrm{O}^{+}}}=4.12 \pm 0.17
$$

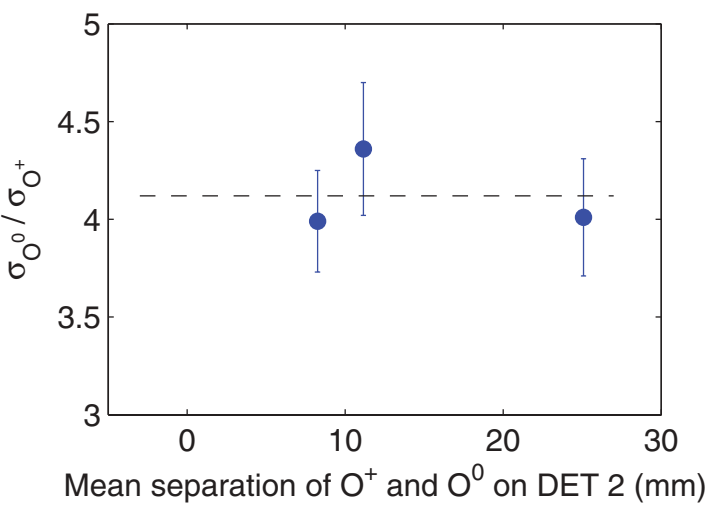

FIG. 4. (Color online) Investigation of a possible variation on detector efficiency across DET 2: the measured ratio of single and double detachment as a function of the mean spatial separation of impact positions of $\mathrm{O}^{+}$and $\mathrm{O}^{0}$ particles.

\section{B. Photoelectrons from single detachment}

The selective detection of electrons from the photodetachment processes is intrinsically more difficult than the detection of neutral and charged photofragments since the electrons arising from photodetachment must be detected on top of a background of similar photoelectrons resulting from ionization of the residual gas (mainly $\mathrm{H}_{2}$ at $\sim 10^{-10}$ mbar). The detection of the heavy fragments $\left(\mathrm{O}^{0}, \mathrm{O}^{+}\right)$are insensitive to this background since they propagate at high speed to DET2 (see Fig. 2), while the ionized species from the residual gas are formed at thermal velocities. Additionally, stray xuv light along the photon beam path may generate signals on the nearby electron detectors (see Fig. 2) due both to photons scattered directly onto the detector and to photoelectrons released from surfaces inside the interaction zone. The background from electrons generated by ion impact onto residual gas molecules is essentially negligible.

More explicitly, the number of fragments produced in a photodetachment reaction $X\left(=0^{0}, \mathrm{O}^{+}\right)$within a single ionphoton crossing can be written as $R_{X}=\sigma_{X} T_{P G 2} N_{\gamma} l_{O^{-}} F$ [see also Eq. (2)] while the number of fragments resulting from collisions with the residual gas is

$$
R_{X}^{g}=\sigma_{X}^{g} n_{g}\left(I_{O^{-}} / e\right) L_{X}^{g} \Delta t_{X},
$$

where $\sigma_{X}^{g}\left(\sim 10^{-15} \mathrm{~cm}^{2}\right)$ is the cross section for detachment in the residual gas, $n_{g}\left(\sim 2 \times 10^{6} \mathrm{~cm}^{-3}\right)$ is the residual gas density, $I_{O^{-}} / e(\sim 1 \mathrm{nA} / e)$ is the rate of ions, and $L_{X}^{g}(\sim 150 \mathrm{~cm})$ is the effective propagation length from which fragments from the process $X$ reach DET2; and last, $\Delta t_{X}(\sim 30 \mathrm{~ns})$ is the temporal range when photodetachment fragments from the ion beam are observed. Similarly, the number of photoelectrons produced from ionization of the residual gas in a single ionphoton crossing is

$$
R_{e}^{g}=\sigma_{e}^{g} T_{P G 2} N_{\gamma} n_{g} L_{e}^{g},
$$

where $\sigma_{X}^{g}\left(\sim 10^{-18} \mathrm{~cm}^{2}\right)$ is the photoionization cross section and $L_{e}^{g}(\sim 1 \mathrm{~cm})$ is the effective length from which photoelectrons are extracted to the electron detectors. For the present experiment $R_{O^{0}} \sim 2 \times 10^{-3}$ and $R_{O}^{g} \sim 1 \times 10^{-4}$, while $R_{e}^{g} \sim 6$. These estimates clearly demonstrate the intrinsic difficulty in photoelectron detection compared to fragment 
detection. With both electrons from photodetachment and from ionization of the residual gas arriving at the electron detectors within $\leqslant 200 \mathrm{~ns}$ after the photon pulse, a maximum of $\sim 2-3$ electrons following each pulse can be tolerated without detector saturation.

Once counting of the electron signal following each FLASH pulse could be achieved, we accomplished photoelectron detection in spite of the high background by requiring coincidence between fragmentation events on DET2 and signal on one of the electron detectors. This method is feasible since the heavy-fragment events on DET 2 can be identified with a low background. A precise evaluation of the contribution from random coincidences was obtained by performing an identical coincidence analysis for uncorrelated data sets where the heavy fragment and the electron signals did not originate from the same ion-photon crossings. Figure 5(a) shows the results of this analysis for $1.6 \times 10^{6}$ ion-photon crossings with $P_{P G 2}\left\langle N_{\gamma}\right\rangle=7.3 \times 10^{11}(3.5 \mu \mathrm{J} /$ pulse $)$, where coincidence was required between neutrals $\left(\mathrm{O}^{0}\right)$ and photoelectrons. A small, however significant, difference between the spectra obtained for real and random coincidences is seen at 40-50 ns. The actual difference between the spectra is shown as the
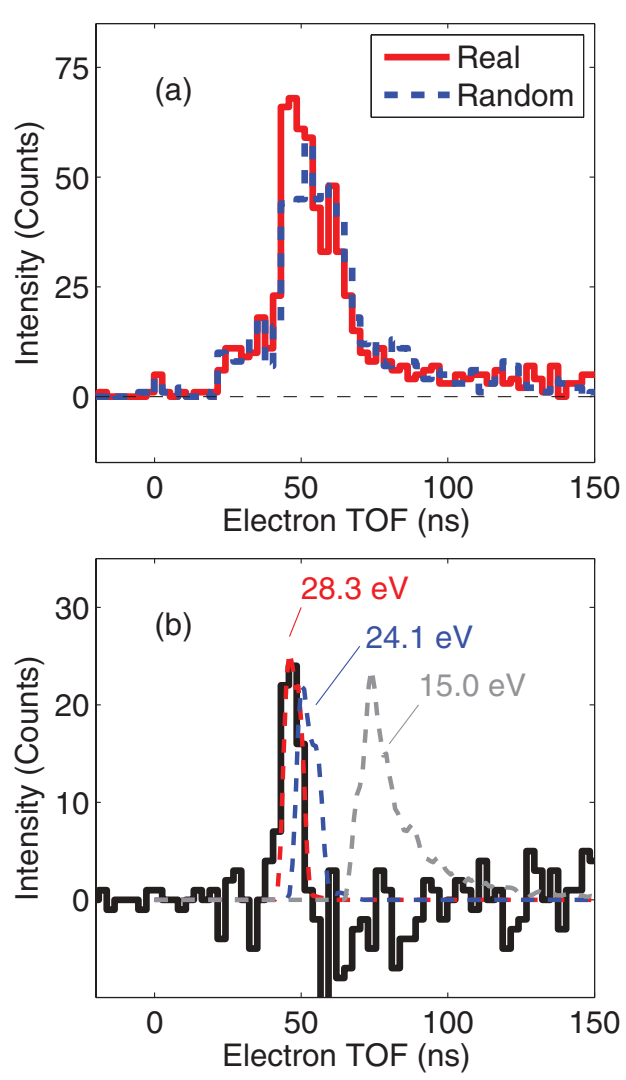

FIG. 5. (Color online) Photoelectron-photoneutral coincidence spectroscopy on single electron detachment from $\mathrm{O}^{-}$at $41.7 \mathrm{~nm}$. (a) Distribution of electron time of flight (TOF) for real (solid red curve) and random (dashed blue curve) coincidences between neutrals $\left(\mathrm{O}^{0}\right)$ and photoelectrons. (b) Difference of the distributions for real and random coincidences (solid black curve) and distributions obtained from Monte Carlo simulations of the experimental conditions for photoelectron energies of $24.3 \mathrm{eV}$ (dashed red curve), $24.1 \mathrm{eV}$ (dashed blue curve), and $15.0 \mathrm{eV}$ (dashed gray curve). solid black line in Fig. 5(b). This figure also displays results of Monte Carlo simulations for electrons created by single detachment reactions from a $1-\mathrm{mm}$-diameter beam of $\mathrm{O}^{-}$at $3 \mathrm{keV}$ at three different photoelectron energies.

A good agreement between the observed spectrum and the results from the Monte Carlo simulations is seen for a photoelectron energy of $28.3 \mathrm{eV}$, corresponding to detachment into the ground state $\left({ }^{3} P\right)$ of the oxygen atom. The electron energy resolution obtained for the spectrometer settings applied in this measurement does not allow us to exclude detachment into the $\left({ }^{1} D\right)$ state. However, the comparison with the other simulations shows that single photodetachment at $41.7 \mathrm{~nm}$ populates neither the ${ }^{1} S$ state nor higher excited states (see Fig. 1).

Since the yield of $\mathrm{O}^{+}$fragments is considerably smaller, a similar analysis of the electron spectrum corresponding to the double detachment channel is not meaningful for the present data. For this reason, the present results do not allow any conclusion on the relative significance of indirect or direct nature of the double detachment reaction.

\section{DISCUSSION}

Recently [26], we investigated photodetachment of the oxygen anion in the uv regime involving the first $\left({ }^{1} D\right)$ excited state of oxygen (see Fig. 2) in an experiment analyzing the kinetic energy and angular distributions of emitted photoelectrons and determining the ratio of ground- and excited-state oxygen atoms after photodetachment at $266 \mathrm{~nm}$. With the present study we have made a first step into investigating the xuv photodetachment of $\mathrm{O}^{-}$by measuring the ratio of single and double detachment for a photon energy far above threshold and also shown the feasibility of photoelectron spectroscopy in this regime using a crossed beams geometry. At a photon energy of $\sim 30 \mathrm{eV}$ we find that single electron detachment, producing $\mathrm{O}^{0}$ atoms in the lowest states $\left({ }^{3} P\right.$ and $\left.{ }^{1} D\right)$, exceeds double photodetachment by a factor of $\sim 4$. For photoelectron spectroscopy (Fig. 5) several changes of the experimental conditions can be expected to increase the statistical quality of the data in future measurements. Thus, the applied ion current $(1 \mathrm{nA})$ can be increased significantly with other ion sources, and the effective repetition rate of the FLASH laser can be increased by allowing a higher number of micropulses than the 50 pulses used presently. Moreover, the background of electrons from the residual gas can be reduced by further improving the vacuum condition below $10^{-10}$ mbar.

The present experiment in fact represents the first direct determination of a ratio of single and double detachment in the xuv regime. The first experimental estimate of a ratio between single and double detachment reactions were done in the case of $\mathrm{He}^{-}$[9] in the uv regime where a ratio of a few permille was reported near threshold. In that case, however, the yield of neutral fragments was determined indirectly from a fit to the yield of $\mathrm{He}^{+}$fragments assuming implicitly that a two-photon process would probe all $\mathrm{He}^{0}$ resulting from single electron photodetachment with similar cross section.

The first pioneering crossed beams studies of photodetachment in the xuv region were carried out on a relativistic beam of $\mathrm{H}^{-}[5,8]$. While these experiments were in fact capable of detecting separately all heavy fragments from the detachment 
reaction $\left(\mathrm{H}^{+}, \mathrm{H}^{0}\right)$, they focused explicitly on the threshold behavior of the double detachment cross section. Moreover, although conceptually universal, the use of relativistic ion beams is in practice limited to studying a few selected species.

In more recent studies of detachment from valence electrons in the xuv regime using synchrotron radiation $[11,16,17]$, the production of neutral fragments from direct detachment was assumed to be insignificant and explicitly not measured with the applied merged beams setups. The present study illustrates that even far above threshold the yield of neutrals arising from single electron photodetachment can be the dominating detachment process. This obviously raises the question of the importance of the single detachment process in the previously studied systems $\mathrm{Na}^{-}$[11], $\mathrm{F}^{-}$[16], and $\mathrm{Cl}^{-}$[17].

The present experiment relies on the use of a fast moving ion beam crossed by an intense photon beam. The directed forward motion of the ion beam allows universal access to all heavy fragments emerging from the detachment reaction including both neutral and charged species, as exemplified with the experimental arrangement shown in Fig. 2. Additionally, photoelectrons can be analyzed near the interaction region. The merged beams experiments using synchrotron radiation facilities [11,12] are capable of measuring precise absolute cross sections over very large photon energy ranges with high resolution. Hence, crossed and merged beams methods are complementary. The strength of the present crossed beams method is to enable a full characterization of the photodetachment processes at selected wavelengths. Indeed, the technique holds the promise of resolving open questions in atomic photodetachment processes studied previously with the merged beams technique. For example, in the regime of inner-shell detachment, the postcollisional recapture processes $[19,24]$ may be characterized in detail by direct observation of $\mathrm{He}^{0}$ or $\mathrm{Li}^{0}$, specifying the detachment pathway by the observation of photoelectrons. For the regime of valence electron photodetachment, the present results highlight the importance of the single detachment process over double detachment. Future detailed coincidence photoelectron spectroscopy on anion beams may experimentally unravel the significance of sequential and simultaneous double photodetachment.

\section{CONCLUSION}

With an exploratory study of the oxygen anion, we have demonstrated how all channels of negative ion photodetachment can be analyzed simultaneously in a crossed beams setup, taking particular advantage of intense free-electron laser sources in the xuv regime. At $41.7 \mathrm{~nm}$, we find single detachment to dominate over double detachment by a factor of $\sigma_{\mathrm{O}^{0}} / \sigma_{\mathrm{O}^{+}}=4.12 \pm 0.17$ and evaluate the absolute cross section for the single detachment channel to $\sigma_{\mathrm{O}^{0}}=(2.1 \pm 0.6) \times$ $10^{-19} \mathrm{~cm}^{2}$. The spectrum of photoelectrons coincident with the neutral fragment indicates that single electron detachment primarily occurs to the lower states of the oxygen atom. The present technique that combines the crossed beams technique with an intense xuv free-electron laser could well be used to complement the successful research on anion photodetachment performed with synchrotron radiation in recent years, since all emerging fragments including neutrals and photoelectrons can be detected simultaneously, allowing in principle a complete characterization of such processes.

\section{ACKNOWLEDGMENTS}

This work has been supported by the Max-Planck Advanced Study Group at CFEL Hamburg and the Max-Planck Society. H.B.P. acknowledges support from the Lundbeck Foundation. We are greatly indebted to the scientific and technical team at FLASH [28,29], in particular the machine operators and run coordinators, being the foundation of the successful operation and delivery of the SASE-FEL beam.
[1] T. Andersen, Phys. Rep. 394, 157 (2004).

[2] P. Dahl, T. Andersen, L. Jodal, N. J. Kylstra, J. E. Hansen, and N. Vaeck, Phys. Rev. A 48, 2844 (1993).

[3] L. H. Andersen, M. J. Jensen, H. B. Pedersen, L. VejbyChristensen, and N. Djuric, Phys. Rev. A 58, 2819 (1998).

[4] K. Fritioff et al., Phys. Rev. A 69, 042707 (2004).

[5] H. C. Bryant et al., Phys. Rev. Lett. 38, 228 (1977).

[6] P. Balling, H. H. Andersen, C. A. Brodie, U. V. Pedersen, V. V. Petrunin, M. K. Raarup, P. Steiner, and T. Andersen, Phys. Rev. A 61, 022702 (2000).

[7] M. K. Raarup, U. V. Pedersen, V. V. Petrunin, P. Balling, and T. Andersen, Phys. Rev. Lett. 85, 4028 (2000).

[8] J. B. Donahue, P. A. M. Gram, M. V. Hynes, R. W. Hamm, C. A. Frost, H. C. Bryant, K. B. Butterfield, D. A. Clark, and W. W. Smith, Phys. Rev. Lett. 48, 1538 (1982).

[9] Y. K. Bae, M. J. Coggiola, and J. R. Peterson, Phys. Rev. A 28, 3378 (1983).

[10] Y. K. Bae and J. R. Peterson, Phys. Rev. A 37, 3254 (1988).

[11] A. M. Covington et al., J. Phys. B 34, L735 (2001).

[12] H. Kjeldsen et al., J. Phys. B 34, L353 (2001).

[13] N. Berrah et al., Phys. Rev. Lett. 87, 253002 (2001).
[14] N. Berrah, J. D. Bozek, G. Turri, G. Akerman, B. Rude, H. L. Zhou, and S. T. Manson, Phys. Rev. Lett. 88, 093001 (2002).

[15] N. D. Gibson, C. W. Walter, O. Zatsarinny, T. W. Gorczyca, G. D. Akerman, J. D. Bozek, M. Martins, B. M. McLaughlin, and N. Berrah, Phys. Rev. A 67, 030703 (2003)

[16] V. T. Davis et al., J. Phys. B 38, 2579 (2005)

[17] A. Aguilar et al., Phys. Rev. A 69, 022711 (2004)

[18] R. C. Bilodeau, J. D. Bozek, A. Aguilar, G. D. Ackerman, G. Turri, and N. Berrah, Phys. Rev. Lett. 93, 193001 (2004).

[19] R. C. Bilodeau, J. D. Bozek, G. D. Ackerman, A. Aguilar, and N. Berrah, Phys. Rev. A 73, 034701 (2006).

[20] N. Berrah et al., J. Electron Spectrosc. Relat. Phenom. 183, 64 (2011).

[21] H. Kjeldsen, F. Folkmann, T. S. Jacobsen, and J. B. West, Phys. Rev. A 69, 050501(R) (2004).

[22] R. C. Bilodeau, N. D. Gibson, J. D. Bozek, C. W. Walter, G. D. Ackerman, P. Andersson, J. G. Heredia, M. Perri, and N. Berrah, Phys. Rev. A 72, 050701(R) (2005).

[23] J. Sandström et al., Phys. Rev. A 72, 034702 (2005).

[24] T. W. Gorczyca, O. Zatsarinny, H. L. Zhou, S. T. Manson, Z. Felfli, and A. Z. Msezane, Phys. Rev. A 68, 050703(R) (2003). 
[25] D. Hanstorp and M. Gustafsson, J. Phys. B 25, 1773 (1992).

[26] C. Domesle, B. Jordon-Thaden, L. Lammich, M. Forstel, U. Hergenhahn, A. Wolf, and H. B. Pedersen, Phys. Rev. A 82, 033402 (2010).
[27] M. Martins et al., Rev. Sci. Instrum. 77, 115108 (2006).

[28] W. Ackermann et al., Nat. Photon. 1, 336 (2007).

[29] K. Tiedtke et al., New J. Phys. 11, 023029 (2009).

[30] H. B. Pedersen et al., Phys. Rev. A 80, 012707 (2009). 